\title{
Walking the Walk to Teach the Talk: Implementing Ancestral Lifestyle Strategies as the Newest Tool in Evolutionary Studies
}

\author{
Steven M. Platek • Glenn Geher • Leslie Heywood • \\ Hamilton Stapell • J. Ryan Porter • Tia Y. Walters
}

Published online: 27 January 2011

(C) Springer Science+Business Media, LLC 2011

\begin{abstract}
The learning of evolutionary theory typically takes place in the classroom or laboratory. Students of these traditional approaches often leave with the notion that applications of evolutionary theory have little bearing on their lives. The Evolutionary Studies Consortium (EvoS; evostudies.org) has been extremely successful in overcoming these barriers and demonstrating the bridges across academic areas that can be created with the principles of evolution as a guide. While this is a fantastic means through which to educate students about the intricacies of evolution, we believe that the full potential of this approach has yet to be realized.
\end{abstract}

\footnotetext{
S. M. Platek $(\varangle) \cdot$ J. R. Porter $\cdot$ T. Y. Walters

Department of Psychology,

Georgia Gwinnett College,

Lawrenceville, GA, USA

e-mail: splatek@gmail.com

J. R. Porter

e-mail: jporter@ggc.edu

T. Y. Walters

e-mail: twalters@ggc.edu

G. Geher

Department of Psychology,

SUNY-New Paltz,

New Paltz, NY, USA

e-mail: geherg@newpaltz.edu

H. Stapell

Department of History,

SUNY-New Paltz,

New Paltz, NY, USA

e-mail: stapellh@newpaltz.edu

L. Heywood

Binghamton University,

Binghamton, NY, USA

e-mail: heywood@binghamton.edu
}

Applications beyond strict academic contexts are still waiting to be mined. Here, we outline an approach that proposes the implementation of a nutrition and physical fitness program, alongside classroom pedagogy, as a means of helping students learn about evolution and how it can be used to increase their own quality of life.

Keywords Health and fitness - Evolutionary health . Ancestral lifestyle

\section{EvoS as a Builder of Bridges across Islands of the Academic Archipelago}

The EvoS Consortium, which is highlighted in this special issue of Evolution: Education and Outreach, provides strong evidence that Darwin's ideas have the capacity to revolutionize modern academia. As David Sloan Wilson (2007) tells us, academia is not really the Ivory Tower of traditional metaphoric usage - it is an archipelago- perhaps an ivory archipelago - comprised of discrete islands whose native inhabitants are either unaware of one another or who are extremely skeptical of one another's intentions. Theatre arts is an island. Business management is an island. Computer engineering is an island. Physical education is an island. Microbiology is an island. Sociology is an island.

One might think that the current movement toward interdisciplinary studies that characterizes modern academia is a step toward solving the archipelago problem. Clearly, interdisciplinary efforts have increased across the landscape of higher education (Tappeiner et al. 2007) with beneficial effects. With that said, traditional interdisciplinary approaches have clear limits. Many typical interdisciplinary kinds of programs, in areas such as Black Studies, Religious Studies, or Women's Studies, for instance, 
address a particular set of content from the perspective of different academic approaches.

EvoS is different (see Carmen et al. 2010). EvoS programs are connected by a shared set of intellectual principles and ideas rather than a shared set of content. The basic ideas of natural selection, sexual selection, and cultural evolution, for instance, can be applied broadly to add insights into such disparate areas as anthropology, entomology, geology, philosophy, and social psychology (see Garcia et al. 2011). While EvoS has provided an infrastructure for expanding the application of evolutionary science in education across disciplines, others have undertaken the idea as well (see Bjorklund and Brown 2008; Geary 2008; Gray 2010).

This landmark approach to higher education, which started as the result of David Sloan Wilson's efforts in Binghamton in 2003, has proven so successful that we now have a consortium of $40+$ schools that have some level of involvement in this approach to undergraduate education. Furthermore, we have received a grant of $\$ 500,000$ from the National Science Foundation (NSF\#0817337) to bolster our efforts and have established a major web presence in evostudies.org, complete with links to online videos of EvoS speakers across institutions, the EvoS blogs, EvoS Journal: The Journal of the Evolutionary Studies Consortium (a peer-reviewed journal dedicated to issues related to evolution in higher education), and a library of curricular resources designed to enhance courses related to EvoS. EvoS has an unprecedented capacity to build bridges across the Ivory Archipelago - and judging by the broad interest in EvoS from colleges and universities across the world, coupled with substantial support from the NSF, we believe this stance is justified.

Ultimately, a strong intellectual approach should be used to the benefit of individuals and society. Given the powerful nature of evolutionary theory in explaining phenomena across islands of the Ivory Archipelago, EvoS has extraordinary potential to lead to positive social and personal applications. Efforts toward this end are under way. Consider the major advances in Darwinian medicine (Williams and Nesse 1991, 1996), for instance, that have taken place in recent years. Evolution is also beginning to be applied in such fields as educational psychology and urban planning.

Given how basic evolutionary principles are to the nature of biological organisms, it should be fundamental to apply evolution to the areas of personal nutrition and physical fitness. While a growing body of scholarship is starting to take this idea seriously, to our understanding, there is not yet a single college course in the United States that is dedicated to the application of evolutionary principles to the topics of nutrition and physical fitness. Additionally, there is not yet a class for which nutrition and physical fitness are utilized as pedagogical tools to aid students in learning how a complete understanding of evolutionary principles can actually increase their quality of life.

Changes are on the horizon. For one, a world-class international conference dedicated to evolutionary applications to nutrition and physical fitness is slated for summer 2011 on the campus at UCLA (www.ancestryfoundation. org). Second, many major approaches to nutrition now explicitly address food in terms of our evolutionary history as a species (Cordain et al. 2002). Finally, the current explosion of the CrossFit ${ }^{1}$ physical fitness program is bringing our understanding of our evolutionary heritage to the forefront of modern physical fitness training. Maybe it is time to start a college class in physical fitness and nutrition from the angle of evolutionary theory.

To address this issue, we have developed a new tool to teach evolutionary principles and reap the benefits for individuals and societies. This approach entails a carefully prescribed physical fitness and nutrition regimen that, based on what we can surmise from anthropology, psychology, biology, physiology, exercise science, and sports psychology, approximates what our ancestors faced on a daily basis and modern hunter gatherer groups undergo daily. This physical fitness and nutrition program, which we are tentatively calling EvoT (pronounced evo-tee; Evolutionary Training), makes use of three basic principles to aid education about evolution while simultaneously increasing student physical health and fitness. First, the students are exposed to evolutionarily inspired physical fitness programming. This programming utilizes the Power Law model for exercise that prescribes workouts that are short in duration but high in intensity (e.g., sprinting) combined with frequent movement at a slow pace (e.g., hiking/walking). The program includes an exercise regimen that is designed to be constantly varied. This constant variance of physical stimulus regularly confuses and challenges the body both muscularly and neurologically and aids in the avoidance of the all too common plateau effect in physical performance. The exercises include movements that are considered functional in nature (e.g., lifting objects as opposed to curling your leg). This form of exercise regimen taps all three major metabolic pathways

\footnotetext{
${ }^{1}$ CrossFit (CrossFit.com) is but one physical fitness outlet that is offering education and training in functional movements that approximate ancestral patterns of movement. Other iterations of such evolutionarily minded physical fitness programs include, but are not limited to: (1) Arthur Devany's Evolutionary Fitness (http://www. arthurdevany.com/); (2) potentially, an even closer approximation, Erwan LeCore's MovNat. The latter involves training seminars where participants are asked to live in the forest and swim, hunt, climb, run over obstacles, as well as forage for some of their own food; and (3) The Primal Blueprint (www.marksdailyapple.com). We utilize the CrossFit methodology for this paper because we are currently using it in our first attempt at a course like EvoT.
} 
for energy expenditure: the phosphagen, the glycolytic, and the oxidative. Second, students are exposed to the Paleolithic diet (http://www.thepaleodiet.com/), which is our best estimation of what our ancestors subsided on. And third, students will be taught why this particular exercise and nutrition program works to aid their weight loss, muscular development, general physical fitness and preparedness, athletic performance, mental acuity, and psychological well-being.

\section{The Next Step in EvoS Evolution: Evolutionary Training}

Evolutionary fitness and physical fitness are two separate terms that, although different, correspond to the ideal healthy Homo sapien. Evolutionary fitness or Darwinian fitness is dependent not only on the number of offspring produced, but also by the differential rate of an increase of genotypes in a population (Roff 2008). Evolutionary biologists often refer to this as genetic representation in subsequent generations. In contrast, physical fitness is the amount of acquired physical attributes a person possesses. According to the U.S. Department of Health and Human Services, there are two aspects of physical fitness. First, health-related fitness, common among those in the general population, is the concentration on the reduction or movement out of disease and/or injury. Second is performance-related fitness which emphasizes the amount of physical training required to achieve a physically demanding objective. The primary purpose is to have the physical capability to accomplish all aspects of a goal while remaining healthy and/or uninjured (Roy et al. 2010). Darwinian fitness and physical fitness are likely closely related: physically fit (that is, healthy, disease-free individuals that can solve physically demanding challenges) would make good mate choices (at least under certain selective pressures). On the other hand, physical fitness and/or prowess might aid Darwinian fitness by making physically demanding behaviors (e.g., mate guarding, protecting offspring and family, acquiring resources) easier and/or more likely. If we take into consideration the expanded evolutionary principles of the Extended Synthesis that will be discussed later in this paper, even more profound may be the question of how physical fitness impacts maternal health and the health of the developing fetus. Recent research has shown the negative effects of maternal diet or environmental carcinogens upon a fetus. On the other hand, what would be the measurable positive effects on fetal and infant health of a generation of women who train through their pregnancies, as has been happening in the generation of women born post Title IX in the U.S., for whom serious athletic training has always been a cultural norm? This question lies beyond the scope of this paper, but points to a crucial research area for future work.

Current physical fitness practices often fail to take our evolutionary heritage into account. For example, it's generally agreed that ancestral humans lived a varied and active lifestyle. Physical exertion was vital to survival, both in food procurement and escape from predation (Cordain et al. 1998). Myriad activities were part of daily life and occurred spontaneously using universal motor recruitment patterns. These include: walking and running long distances, lifting, carrying, climbing, stretching, leaping, and virtually any other behavior that may have been needed in order to obtain food and/or safety (Abuissa et al. 2005). Natural selection favored functional movements ${ }^{2}$ that would have better equipped our ancestors to do these things.

It would have behooved our ancestors to be as efficient as possible; any wasted energy would have exponentially decreased the likelihood of survival and reproduction. An example of efficient movement might have taken place during long-distance hunts. In Homo erectus, "the earliest species in the genus Homo whose body mass and locomotor morphology resembled that of modern humans", walking at optimal speeds would have used up $64 \%$ of their daily energy expenditure. Running continuously at optimal speeds would have used $84 \%$. As a result, it might have been more beneficial, at times, for our ancestors to move more slowly (i.e., when traveling from campsite to campsite, while hunting/gathering), with intermittent bursts of high-intensity running (i.e., when they spotted prey or a predator; Eaton and Eaton 2003). Today, running/walking 5Ks, marathons, ultra marathons, and competing in track and field (e.g., sprinting) are done primarily for exercise and sport but are quasi-parallel to what our ancestors might have had to do on a regular basis simply to ensure their survival.

The functional movements that aided our ancestors are just as efficient for modern humans today. These movements are compound yet irreducible and work from core to extremity. Many gyms and fitness centers are very well equipped with machines that work isolated muscle groups (e.g., leg extension, biceps curl, triceps push down), but the lack of compound or multi-joint movements make this type of workout regime, especially at high loads, purely unnatural to humans. The various lifts and movements implemented by CrossFit (and other evolutionarily inspired physical fitness programs, see above) serve functional

\footnotetext{
${ }^{2}$ Functional movements are defined as movements that 1) recruit more than one group of muscles, 2) recruit primarily large muscle groups (e.g., gluteus complex, hip flexor and iliopsoas complexes), 3) work from core to extremity, and 4) are efficient at moving large loads long distances quickly, if needed.
} 
purposes that mimic motor recruitment patterns that are found in everyday life and endorse General Physical Preparedness (GPP), or physical fitness, that no isometric machine could come close to matching. That is, the exercise prescription put forth by CrossFit and CrossFit.com prepares individuals to be physically prepared to tackle physically related obstacles even if those obstacles are unknown to the individual. For example, one might never climb his/her way across a river via a tree bridge. The fitness prescription set forth in EvoT should prepare an individual to (1) be able to tackle that obstacle and (2) be able to do it better than if the individual had trained isolated muscle groups.

\section{Example Exercises}

The dead lift (see Glassman 2003a; see Fig. 1) is one of, if not the most important and functional exercises available to move heavy weight. This movement is unmatched in its simplicity while exceptional in its capacity for increasing full body strength. Unfortunately, this exercise is seldom seen in the traditional fitness clubs because of fear of injury. If executed correctly, however, the dead lift is undeniably the safest way to lift heavy loads off the ground. Our ancestors would have used this lifting technique to pick up large and/or heavy objects such as stones or children, to drag animal carcasses, and to move materials for shelter construction (Eaton and Eaton 2003; Eich 2009). Today, this modus operandi is used in much the same way: to lift heavy objects from the ground, such as a bag of dog food, luggage, and moving furniture and heavier materials used in home maintenance. In contrast, a biceps curl serves littleto-no functional purpose. Our ancestors would not have slowly curled a gazelle leg to the mouth for consumption. This is an isolated muscle building exercise used to develop "mirror muscles" (musculature that looks good as one poses in front of a reflective source as opposed to muscles that solve physical problems).

Another quintessential functional movement is the squat (see Glassman 2002; see Fig. 2). This one movement is vital to a person's overall well-being and can assist in advancing an individual's physical fitness by allowing the back, hips, and knees (i.e., multi-joint, multi-muscle) to remain reliable and functioning well into one's elderly years. Squatting is likely to be the technique that is used most frequently, and when combined with other techniques, it increases power and one's ability to pick up a greater amount of weight, faster. Ancestrally, the squat probably pervaded everyday life. From standing up, semi-squatting in preparation for a jump, a variation of a one-legged squat used when climbing trees, up sheer cliffs, to squatting when lifting very heavy objects like logs and larger, heavier animals, this movement was a regular activity $\left(\mathrm{O}^{\prime}\right.$ Keefe
Fig. 1 The deadlift. Image provided courtesy of CrossFit. com, CrossFit, Inc
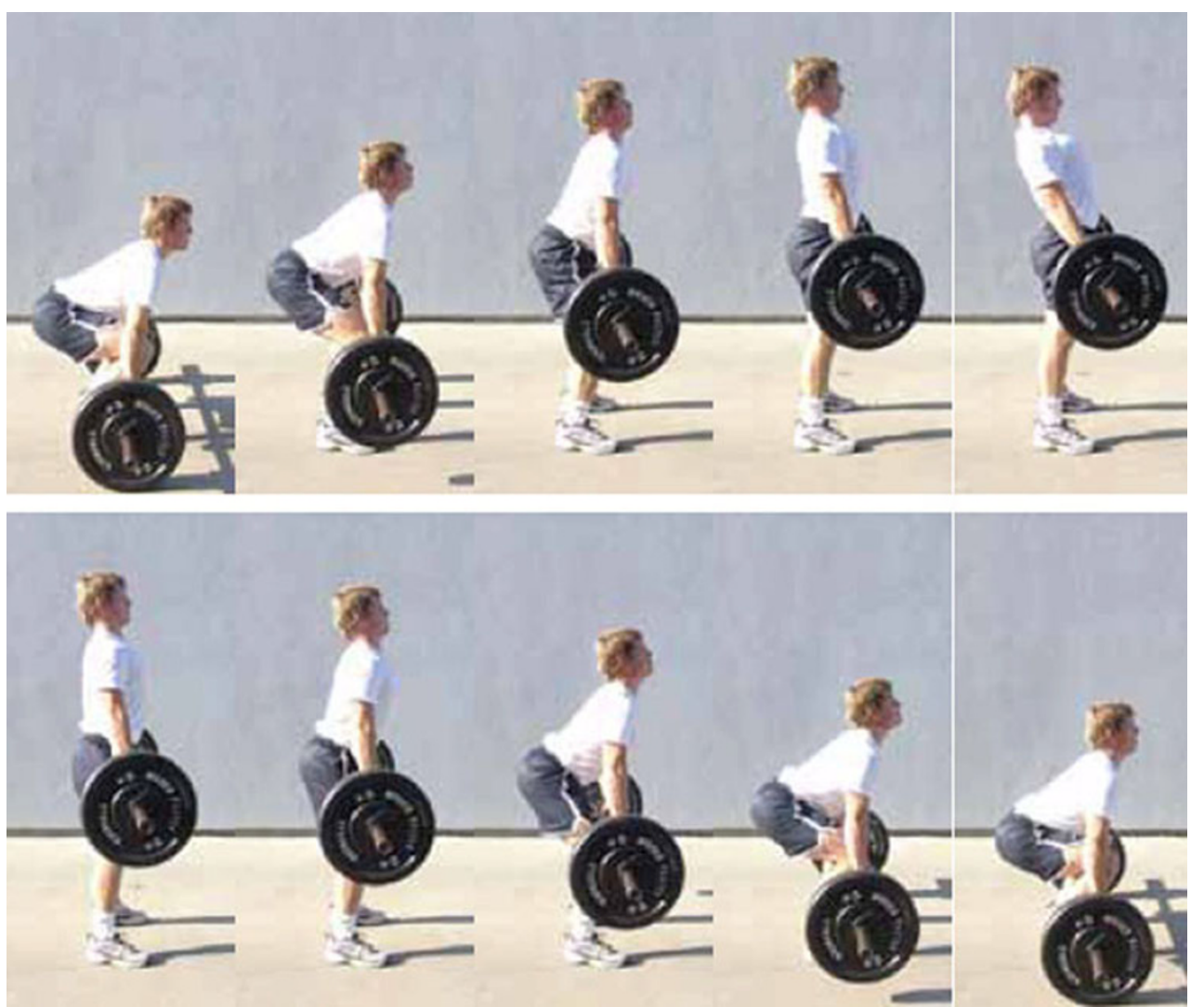
Fig. 2 The air squat. Image provided courtesy of CrossFit. com, CrossFit, Inc
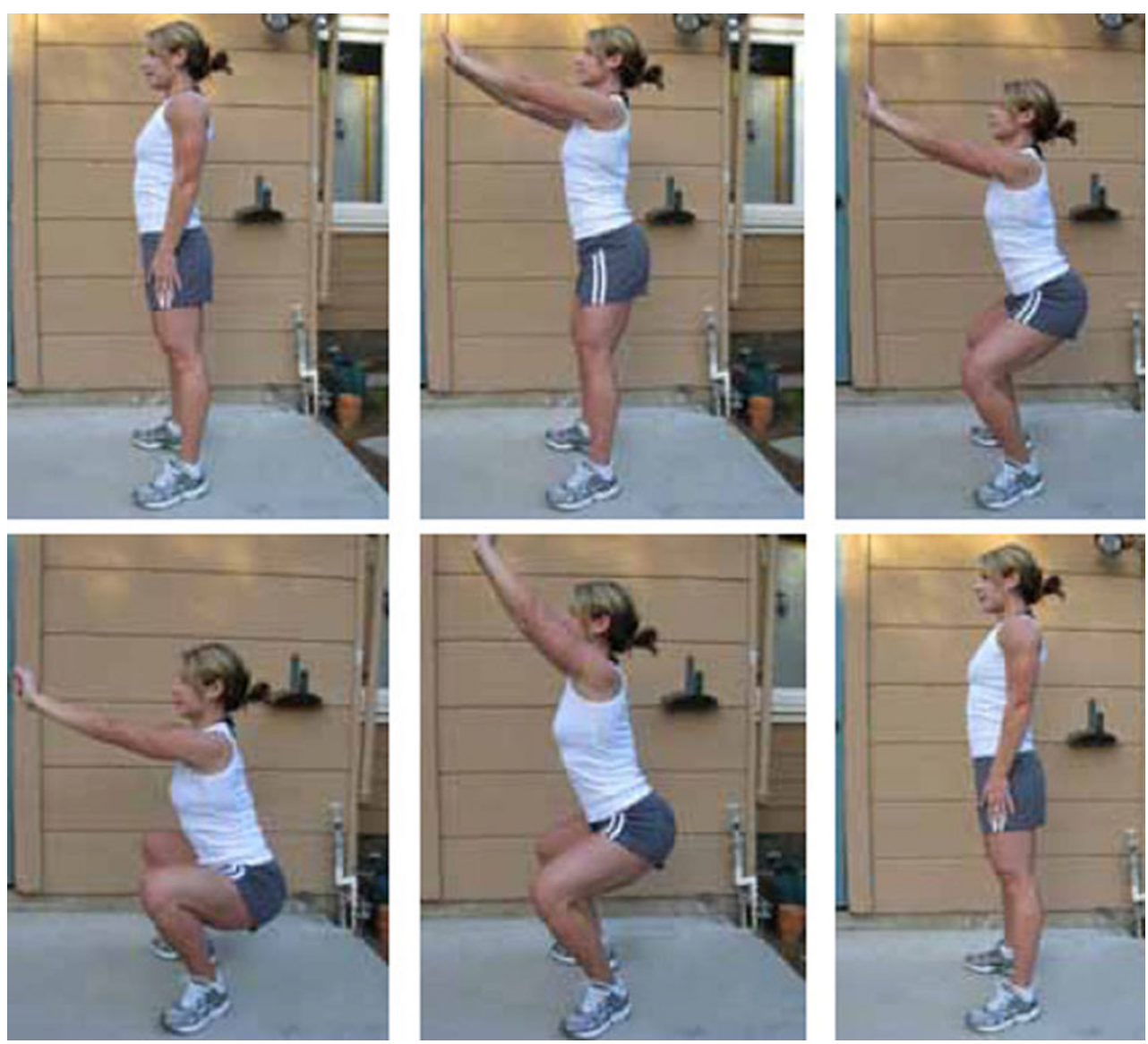

and Cordain 2004a). Moreover, the bottom position of the squat is the stance used by many hunter-gatherer societies and young children to excrete solid waste, which flies in the face of exercise scientists' commonly held notion that going low in a squat can be detrimental to the knees. Even when sitting, the bottom of a squat is the natural position, a position occupied by young children frequently, and a position that is unavoidable should you try to get up off of the ground from your back. Although times have changed, these same basic movements are imperative to humans today. In comparison, a leg extension on a nautilus machine is nonfunctional-it utilizes one set of isolated muscles and executes the movement in an unnatural fashion, and has no use in the everyday world.

The ability to get something from the ground to the shoulder would have been useful to our ancestors (e.g., carrying hunting gear, an injured relative, or a small dead carcass). The most efficient way to do this is the power clean, and for heavier objects, the squat clean (see Glassman 2003b; see Fig. 3). This compound movement combines a deadlift with a powerful shoulder shrug and a drop into a squat to get beneath the object, then bringing it to rest on the clavicles or shoulders (trapezius). This is a movement most men and women would recognize from the simple gesture of picking up a heavy object and relocating it to an elevated location.
A final movement that occurs with regularity in CrossFit, and most functional fitness programming, yet may not have the recognition of some of the more common exercises, is the kettle bell swing (see Glassman 2004). To perform this movement, one stands straight with their legs shoulder-width apart. The individual then leans forward at the waist slightly and bends the knees, going into a semisquat. Keeping the back arched and face forward, the person then grasps the kettle bell. Rocking slightly he/she swings the weight with both hands in between the legs. Then, using the power of a thrust originating from the hip, quadriceps, glutes, and lower back, the individual launches the weight forward than up overhead. Our ancestors may have used a similar technique while shoveling, for example during the burying of the dead (Bar-Yosef Mayer et al. 2009). Even though today a between-the-legs motion is not used while digging, the kettle bell swing still engages muscle groups that are needed for this task. Additionally, kettle bell swings strengthen the spinus erectus muscles of the lower back that are engaged in the above-mentioned essential exercises - dead lift, squat, and clean - to a high degree, thus further strengthening the core as opposed to extremity muscle groups. Surprisingly, however, training using the core to extremity approach does actually accentuate the development of muscles such as the biceps 
Fig. 3 The clean squat. Image provided courtesy of CrossFit. com, CrossFit, Inc
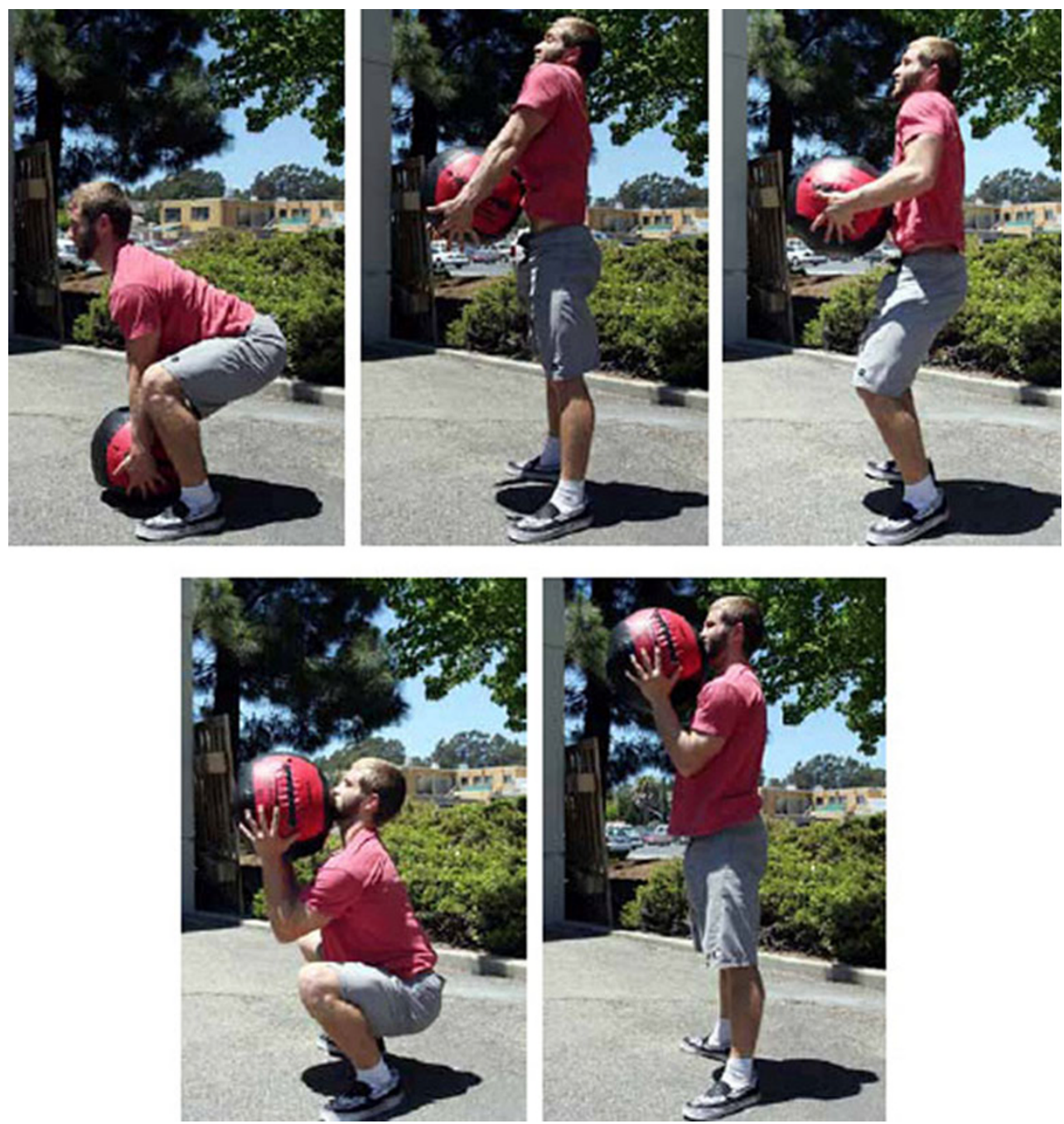

brachii. Platek has surmised that training in a way that is functional and ancestral will not only produce optimal physical fitness and general physical preparedness but also produce optimally designed bodies (e.g., accentuate waist-tohip ratio in women and shoulder-to-hip ratio in men). This hypothesis is currently being tested.

Conventional physical fitness standards must be challenged with a neo-regimen that draws from movements that are basic, functional, and, thus, ancestral in form. What we would like to advocate is a functional modification to physical fitness that not only makes one look good naked but supports the health and well-being of the individual throughout the course of his or her life. With this thinking, CrossFit prescribes the most applicable physical fitness program. Using the CrossFit methodology, ten primary modalities can be trained and improved: cardiovascular/respiratory endurance, stamina, strength, flexibility, power, speed, coordination, agility, balance, and accuracy (Glassman 2010). With such evolutionary and functional training, lives will be changed to not only increase longevity but also enhance their quality.

\section{Nutritional Prescription: The Paleolithic (Caveman) Diet}

The second principle to aid education about evolution while simultaneously increasing student physical fitness and health pertains to nutrition. Our approach covers the evolution of human eating patterns and then relates those patterns to health and physical fitness today. More specifically, we examine what humans ate for the approximately 2.5 million years prior to the Neolithic Revolution (the invention of agriculture) and how that way of life can serve as a guide for maximizing well-being in our modern world. Rather than simply emphasizing the memorization of information, this approach focuses on the daily implementation of nutritional strategies based on our evolutionary past. Thus, the main objective is for students to acquire a thorough understanding of the basic processes of evolution as they relate to human health and to develop the nutritional habits to improve physical fitness and overall well-being.

After completing the nutritional component of EvoT, students should be able to accomplish the following three 
educational goals. First, they should be familiar with the basic concepts of evolution, including natural selection, sexual selection, adaptation, and cultural evolution. Second, students should understand the major milestones in human evolutionary development as they relate to nutrition over the past 2.5 million years. Third, participants in EvoT should be able to construct a weekly eating plan using "Paleolithic" food sources that were commonly available prior to the invention of agriculture. The combination of these three goals will allow students to experience for themselves the power and usefulness of evolutionary principles.

The nutritional regimen we prescribe as part of EvoT is commonly referred to as the Paleo Diet. This diet involves eating only foods that we think our ancestors $(>10,000$ years ago) would have had access to, thus avoiding foods that have flourished under human agricultural evolution and domestication, and foods that are processed and that huntergatherers would not include in their regular diet. This approach is centered on the idea that the genus Homo has been evolving for more than two million years, yet humans have only been farming for the past 10,000 years. And, more to the point, modern humans are almost genetically the same as our Paleolithic ancestors (Lindeberg et al. 2003). As a result, our bodies have been designed to eatand are accustomed to eating - a hunter-gatherer diet, not the products of the Neolithic Revolution. In fact, humans lost both stature and strength with the invention of agriculture (Richards 2002).

The Paleo Diet we prescribe includes: fruits and vegetables, nuts and seeds, meat and fish, and eggs. At the same time, it avoids recent additions to the human diet, such as wheat, rice, corn, legumes, potatoes, and dairy products. In addition, foods that have been introduced in the past 100-150 years are strictly excluded (e.g., processed foods). These products of industrial food production include: refined sugars and flours, processed and packaged foods, fast food, hydrogenated and processed vegetable oils, artificial flavors and sweeteners, and soft drinks. As a species, we are simply not adapted to eat these foods. This Paleo or ancestral human diet is obtained from historical and ethnographic studies of modern-day hunter-gatherers and from archeological finds and anthropological evidence. Our focus, however, is not just on what is eaten but also on how it is produced.

The idea is to select foods that most closely approximate their "natural" or "original" state and to avoid industrialized food products. For example, freerange chickens, and their eggs, are closer to wild chickens than is conventionally, group-raised poultry. Likewise, grass-fed, free-range beef and wild-caught fish should be chosen whenever possible over their conventional alternatives. The same goes for produce. Locally grown, seasonal, organic fruits and vegetables are preferable to industrially produced and genetically modified food. It has only been for the last 100 years that we have been spraying chemicals on our crops and giving our livestock drugs. In all of the examples above, the non-conventional choice is more nutritious and closer to what our ancestors ate (Lindeberg 2005).

In this sense, there is a clear link between the current discussions of sustainable food production in localized contexts, the "locavore" movement, local economic revitalization movements such as the Business Alliance for Local Living Economies (BALLE), and the changes we are proposing here. As the evidence becomes undeniable that current globalized practices of food production with their massive scale and thousands-mile long supply chains are unsustainable, emphasis has returned to local food production economies. These small-scale economies center around principles of free-range meat and organic farming, both implicitly and explicitly addressing the ethical, ecological, and economic problems with factory farming - a major part of which is their distortion of organisms' evolutionary heritage. As Michael Pollan writes, "American factory farm $[\mathrm{s}]$. . . are places where the subtleties of moral philosophy and animal cognition mean less than nothing, where everything we've learned about animals at least since Darwin has been simply . . . set aside". From pigs and cows that are fed unnatural diets to fatten them faster and given antibiotics to mitigate the resulting health problems to chickens that turn to cannibalism due to their unbearable cramped conditions, the factory farm is a site of cruelty that utterly disregards how an animal's evolutionarily developed physiology actually functions. Problems related to the sustainability of our current food production practices function on many levels, from the impact of pesticides and fertilizers on ecosystems to the impact of factory farming on animal physiology to the impact of all of these on human health.

Not only are agriculture and industrial food production quite new (given our evolutionary perspective), there is also a growing body of literature that links dairy and grain consumption with diseases like rheumatoid arthritis, type 2 diabetes, Crohn's Disease, multiple sclerosis, irritable bowel syndrome, and some forms of cancer (Lindeberg 2010). In addition, the "lipid hypothesis" of cardiovascular disease, which usually blames meat and saturated fat consumption, is increasingly coming under scrutiny (O'Keefe and Cordain 2004b). Following the Paleo Diet can improve, and possibly even reverse, these "diseases of civilization" (Lindeberg et al. 2003).

The Paleo Diet also calls for sporadic eating. Today, this is commonly referred to as intermittent fasting. The idea behind occasionally limiting caloric intake is simple. When food was plentiful, our ancestors ate more than they needed (and stored the excess as fat). When times were scarce, they 
survived on fat stores. This random or "non-linear" eating pattern appears to confer several advantages, including decreasing insulin resistance, giving the digestive system time to rest, and allowing the body an opportunity to heal itself (Varady and Hellerstein 2007). Intermittent fasting is achieved by reallocating calories from one meal to another (for example, by forgoing dinner and then eating more the following day), rather than by reducing the total number of calories consumed in a day or in a week. This is in contrast to calorically restricted diets in which individuals simply reduce the total number of calories by $30 \%$ from their daily caloric requirements. There are several hypotheses regarding how generalized caloric restriction positively impacts longevity ranging from alteration of glycolysis in mitochondria to simply lowering Body Mass Index (for review see Sinclair 2005).

The benefits of following a Paleo Diet are myriad (Cordain et al. 2005a, b). In addition to avoiding the "diseases of civilization" mentioned above, students trained in the EvoT nutritional approach likely will have lower blood pressure, maintain a stronger immune system, become physically stronger, and have improved hormone regulation (Frassetto et al. 2009). They will also have more sustained energy and have better control over insulin production and blood sugar levels (Lindeberg et al. 2007). With increased insulin control, participants will be able to manage their body weight more effectively. Given the dramatic rise in obesity - and the accompanying health care costs associated with this epidemic - over the past two decades, this is a significant benefit to both the individual and society as a whole. It is only through a greater appreciation of human ancestral eating patterns and evolution that this kind of change can take place.

In short, the EvoT approach to nutrition both educates students about evolution and optimizes their health and fitness. For us, it is a two-way street. Teaching the development of human nutrition serves to demonstrate many of the processes of evolution, while exploring the principles of evolution helps guide and inform our dietary choices today. Here, as in the other two principles of the program, our goal is to provide a bridge across academic disciplines and to bring evolutionary theory directly to bear on people's lives in a positive and productive way. ${ }^{3}$

\footnotetext{
${ }^{3}$ While EvoT will strive toward educating students about the Paleolithic-like diet as a means of moving students away from processed, modern foods that confer less nutritional value, advanced iterations of EvoT might include prescriptions for optimizing intake with respects to quantity. In other words, although a transition to an unrestricted Paleo-like diet will likely improve the health and fitness of many people, a more careful dialing in of optimal quantities may further enhance the health and fitness benefits, as well as provide an additional fruitful area for evolutionary research.
}

\section{Enlightenment: Does the Program Work for the Students?}

The third principle of EvoT is that students in this program will be taught/made aware of how and why this particular exercise and nutrition regimen works to aid their weight loss. They will also be taught about the program's potential influence on: muscular development, general physical fitness and preparedness, athletic performance, mental acuity, and psychological well-being. This pedagogical task is being undertaken by collecting data on the students while they are engaged/enrolled in the program.

Does it work? We don't know yet. However, we have outlined a possible means with which educators can implement and test the principles in order to answer that question; this is already underway at one of our institutions. It's important to note that EvoT does not necessarily have to be a course listed in the Department of Physical Education or Exercise Science, although it may very well reside in either one or be cross-listed. EvoT has the breadth to allow it to be taught in any discipline that embraces the EvoS methodology, for example: psychology, biology, anthropology, and political science. EvoT is not simply a course about exercise. EvoT is designed to be a course about utilizing exercise and nutrition regimens to teach people about evolutionary science and how it applies to them, here and now!

One of us (SP) has begun to implement EvoT in a course at Georgia Gwinnett College (GGC) as a pilot investigation of the idea. Here is an outline of how an existing course on evolutionary psychology has been modified to include an EvoT component. In addition to the general requirements of the course that include examinations and a research paper/ presentation, the instructor has also assigned a "personal laboratory/lifestyle change" project. This project is designed to get students thinking about the role evolution plays in their everyday life by asking them to change one thing they do to be in line with EvoT principles. Students are instructed on day one about the objectives of the project: they will be collecting data on themselves using personal diaries; they need to track a set of dependent variables that we believe EvoT will positively influence (e.g., psychological well-being, concentration, sleep patterns and quality, energy level, and mood), as well as make any other notes about their daily lives that they feel might be important (open-ended). They are then told that, after four weeks of closely monitoring their dependent variables, during a baseline period, they are going to be given a list of behaviors they can choose to modify in their lives (for a second four weeks). This list includes: start an evolutionary/ functional fitness program, change their existing fitness program to one that is more functional in nature, change their diet to Paleo, walk an extra 1,000 (or more) steps per day, and/ or change one thing about their diet (e.g., eliminate dairy or 
grains). In other words, the students are not required to revamp their entire existence. Rather, they simply have to make one adjustment in their behavior in line with EvoT. The change they select can be drastic, such as eating a strict Paleo diet or less drastic, such as increasing the number of daily steps by 1,000 . The students are instructed/ encouraged to maintain their new behavior for four weeks and continue to track the dependent variables and personal feelings. At the end of the eight-week session, students turn in their diaries and give a presentation on their experience. The diary data will also be analyzed in mass, and in typical EvoS fashion, the data are then presented to the class in combination with a traditional lecture about evolutionary fitness and nutrition and what the data mean. This prescription for executing an EvoT program can be executed at any college and in almost any course that finds itself in line with an EvoS theoretical perspective.

While the program above is only $50 \%$ executed at time of submission, we do feel that this program is valid and has worked to demonstrate the value of an evolutionary or ancestral lifestyle to our students. Evidence of this comes from data we have collected on a local set of athletes that have altered their training and dietary regimens to an ancestral lifestyle strategy. Because the data being collected in the gym environment is not as standardized, this data is qualitative, case study in nature, but we do think it shows the effects of such alterations in behavior.

Case 1: RP. RP is a 23-year-old male who started working out in April of 2010. After his first workout, he was literally physically debilitated and could not complete the workout. He joined a local CrossFit Affiliate Gym (CrossFit Gwinnett) and began to learn about functional fitness, ancestral living, and Paleolithic eating. In a few short months of living this way, RP has lost almost 30 pounds of fat and has gained strength rapidly. He increased his max deadlift by over 175 pounds! More importantly, RP has learned the essence of EvoT through its ability to instill general physical preparedness. He now reports that his general daily activities such as walking across campus, gardening, and carrying groceries are simple tasks, while previously, they were challenging.

Case 2: JJ. JJ is a 47-year-old male who had been working out for several years prior to embracing an EvoT/ ancestral lifestyle. JJ is a vegan, which makes transitioning to a Paleolithic diet challenging, and he chose to stay with his Vegan eating habits. He joined CrossFit Gwinnett in May. Since joining and learning the basics and importance of functional weightlifting, he reports experiencing an increase in cardiovascular function, he has increased in strength (e.g., deadlift has gone up almost 100 pounds), increased his flexibility to where he is now able to execute full range of motion in his knees, and he also reports a general heightened sense of well-being. JJ has also experienced an increase in GPP as he explains:

"I was driving along and wham there goes my tire! Shit! I immediately reverted back to my prior self thinking this is going to really suck. Then, it dawned on me, I've been training for this. I got out of my truck and changed the tire. Sure I was a bit sweaty, this is Georgia in the summer, but I was not winded, as I would certainly have been previously. I owe this to the functional training I'd been doing for the few months previously."

Case 3: TW. TW is a 29-year-old female who has a long history of athletics and working out. She joined CrossFit Gwinnett in April and started the Paleolithic diet in June. TW has also experienced drastic changes in her strength and cardiovascular capacities. She has also changed in appearance and regularly reports that people are asking about how she "got so fit and in shape so quickly".

Case 4: AK. AK is a 29-year-old female. She has an extensive athletic background and has worked out for most of her adult life. She was first introduced to functional fitness and EvoT in January. She had reported experiencing a plateau - she felt like she had stalled out and could not lose any more weight or "tighten up" any further. This was disappointing to AK. She decided to give CrossFit and an $80 \%$ Paleolithic diet (80\% Paleo, 20\% whatever she likes) a try. AK had also just started a new job where she was forced to buy new business clothing that was appropriate for the office. After just one month of starting the EvoTbased lifestyle change, AK reported that her new clothes did not fit her on her first day of work: they were too big on her! At about the same time AK discovered that she was pregnant with her first child. She was disappointed that she might not be able to continue her new fitness and nutritional regimen, but her doctor assured her that she could continue as long as she felt up to it (turns out this is very much in line with EvoT and ancestral living because hunter-gatherer moms maintain their pre-pregnant activity levels and eating patterns). AK did not waiver from the program for all 41 weeks of her gestation. She experienced an unparalleled benefit from the program with respect to her pregnancy. She gained only 21.5 pounds, and the baby measured perfect for his development, so AK was never underweight. She was stronger than ever during her pregnancy, still being able to execute moves such as the dead lift and the pull-up. She even ran on occasion. Now, 6 weeks post-partum AK has maintained her EvoT activity and has lost all but 2 pounds of her pregnancy weight and has regained strength to pre-pregnancy levels as if she had never missed a step.

While lacking in detail, here, each of these cases outlines the very premise of EvoT: teach a person about ancestral 
living (physical fitness and nutrition) and allow them to reap the rewards of good health and psychological wellbeing. In the classroom, this process is brought full circle pedagogically to teach the students about why and how they are feeling different biochemically, physically, structurally, and psychologically. In fact, at the time of submission, early data from the first EvoT iteration are rolling in. In addition to the many anecdotal reports of general increases in well-being, the data are showing that people improved physically during the EvoT period relative to the baseline period. To quote one student:

"[Physical] exercise and nutrition were never very
interesting to me. I subscribed to an everything in
moderation mantra, but this class has taught me that
we were made to move and eat in a specific way and
that if we move and eat in a way that is consistent with
our evolutionary and biological roots we can experi-
ence health and fitness. This is awesome! I'm in the
best shape of my life and I am sorry that I just
discovered this way of living at the age of 41 ; I've
already started to change things at home so that the rest
of my family can experience the same benefits I have"

\section{Discussion}

By coupling sound evolutionary education and actively placing students in a position to "walk the walk" of our evolutionary ancestors, the students will not only reap the rewards of ancestral lifestyle changes (better physical and psychological well-being). They also gain a deeper and more personal understanding of evolution as it applies to humans and to themselves. Interestingly, the pedagogical part of EvoT is minimal. The quote above demonstrates the almost common sense nature of this teaching procedure: prescribe the physical fitness and/or nutritional change while continuing to teach about how evolution works, and the benefits as well as the link between the two becomes glaringly apparent to the students. This approach neatly dovetails with the ecological awareness called for in Massimo Pigliucci's Extended Synthesis and the basic premise of EvoS. The idea is that evolutionary principles are highly relevant to the lives of modern humans and can be applied in positive ways. The Extended Synthesis begins to rectify the nearly exclusive focus on natural selection, gradualism, and population genetics in evolutionary biology's "modern synthesis." From developing areas such as epigenetics and Evo Devo, to definitional concepts such as phenotypic plasticity, evolvability, and niche construction, the Extended Synthesis emphasizes that there are many, in John Odling-Smee's words, "co-causal process[es] in evolution". We argue that EvoT might be another example of the
Extended Synthesis's expanded approach, bringing together research areas in a new and integrative way.

While exercise physiologist Andrew Hatchett has already implemented the first CrossFit course to be offered at a university as part of the physical education curriculum at the University of Louisiana, he does not include evolutionary theory as part of his emphasis. The EvoS consortium is uniquely situated to do so. As an extension of these ideas, New Paltz and Binghamton are concurrently working to create official courses in the curricula that integrate the academics of their existing EvoS programs with the Athletics/Recreational programs on campus. This course, which will serve as a formally institutionalized version of the program currently underway at Georgia Gwinnett College, will be a significant step in the application of evolutionary theory to the everyday lives of individuals.

\section{References}

Abuissa H, O' Keefe Jr JH, Cordain L. Realigning our 21st century diet and lifestyle with our hunter-gatherer genetic identity. Dir Psychiatry. 2005;25:SR1-SR10.

Bar-Yosef Mayer DE, Vandermeersch B, Bar-Yosef O. Shells and ochre in middle paleolithic Quafzeh Cave, Israel: indications for modern behavior. J Hum Evol. 2009;56:307-14.

Bjorklund DF, Brown RD. Physical play and cognitive development: integrating activity, cognition, and education. Child Dev. 2008;69:604-6.

Carmen R, Dillon H, Geher G. History, biology, and politics neatly intertwined: Lee Dugatkin's Newest Work as an Exemplar of an EvoS Education. EvoS Journal: The Journal of the Evolutionary Studies Consortium 2010;2(2):67-71.

Cordain L, Gotshall RW, Eaton SB, Eaton III SB. Physical activity, energy expenditure and fitness: an evolutionary perspective. Int J Sports Med. 1998;19:328-35.

Cordain L, Eaton SB, Sebastian A, Mann N, Lindeberg S, Watkins $\mathrm{BA}$, et al. Origins and evolution of the Western diet: health implications for the 21st century. Am J Clin Nutr (American Society for Nutrition). 2005a;81(2):341-54.

Cordain L, Eaton SB, Sebastian A, Mann N, Lindeberg S, Watkins BA, et al. Origins and evolution of the Western diet: health implications for the 21st century. American Journal of Clinical Nutrition (American Society for Nutrition). 2005b;81(2):341-54.

Eaton SB, Eaton SB. An evolutionary perspective on human physical activity: implications for health. Comp Biochem Physiol Part A. 2003;136:153-9.

Eich P. The Dead elk and the crossfit question. The CrossFit Journal. 2009. Retrieved from http://library.crossfit.com/premium/pdf/ CFJ_Eich_DeadCowDrag.pdf? $=1281819323 \& \mathrm{~h}=\mathrm{a} 87 \mathrm{~b} 5$ d81ē̄ bf445cd75100856e99f703.

Frassetto LA, Schloetter M, Mietus-Synder M, Morris Jr RC, Sebastian A. Metabolic and physiologic improvements from consuming a Paleolithic, hunter-gatherer type diet. European Journal of Clinical Nutrition. 2009;63(8):947-55. The Health Benefits of Paleocuisine. Science 317 (5835): 175. July 13, 2007.

Garcia J, Geher G, Crosier B, Saad G, Gambacorta D, Johnsen L, Pranckitas. The interdisciplinary context of evolutionary approaches to human behavior: a key to survival in the ivory archipelago. Futures in Psychology. 2011. 
Geary DC. An evolutionarily informed education science. Educ Psychol. 2008;43:279-95.

Glassman G. Squat clinic. The Crossfit Journal. 2002. Retrieved from http://library.crossfit.com/premium/pdf/SquatClinic_Dec02.pdf? $\mathrm{e}=1281823072 \& \mathrm{~h}=83 \mathrm{ad} 6 \mathrm{da} 25006824 \mathrm{cbecd} 5 \mathrm{ec} 352 \mathrm{~b} 734 \mathrm{ba}$.

Glassman G. The clean. The Crossfit Journal. 2003;(11), Retrieved from http://library.crossfit.com/premium/pdf/11_03_The_Cleanpdf? $\mathrm{e}=1281823298 \& \mathrm{~h}=50 \mathrm{bdb} 6 \mathrm{c} 5885372 \mathrm{f} 720089 \overline{8} \mathrm{e} 1063 \mathrm{e} 2 \overline{4} 42$.

Glassman G. The Deadlift. The Crossfit Journal. 2003;(12), Retrieved from http://library.crossfit.com/free/pdf/12 03 Deadlift.pdf

Glassman G. The Kettlebell swing. The Crossfit Journal. 2004;(25), Retrieved from http://library.crossfit.com/free/pdf/25_04_kettle bell_swing.pdf.

Glassman G. CrossFit Level 1 Training Guide. The Crossfit Journal. 2010. Retrieved from http://journal.crossfit.com/2010/05/crossfitlevel-1-training-guide.tpl\# login

Gray P. The evolutionary biology of education: How our huntergatherer educative instincts could form the basis for education today. EVOO, x, xxx-xxx. 2010.

Lindeberg S. Palaeolithic diet ("stone age" diet). Scandinavian Journal of Food \& Nutrition. 2005;49(2):75-7.

Lindeberg S. Food and Western disease: health and nutrition from an evolutionary perspective. Chichester, U.K.: Wiley-Blackwell; 2010.

Lindeberg S, Cordain L, Eaton SB. Biological and clinical potential of a Paleolithic diet. J Nutr Environ Med. 2003;13(3):149-60.

Lindeberg S, Jönsson T, Granfeldt Y, Borgstrand E, Soffman J, Sjöström K, et al. A Palaeolithic diet improves glucose tolerance more than a Mediterranean-like diet in individuals with ischaemic heart disease. Diabetologia. 2007;50(9):1795-807.
O' Keefe Jr JH, Cordain L. Cardiovascular disease resulting from a diet and lifestyle at odds with our Paleolithic genome: how to become a 21 st-century hunter-gatherer. Mayo Clin Proc. 2004a;79:101-8.

O'Keefe JH, Cordain L. Cardiovascular disease resulting from a diet and lifestyle at odds with our Paleolithic genome: how to become a 21stcentury hunter-gatherer. Mayo Clin Proc. 2004b;79(1):101-8.

Richards MP. A brief review of the archaeological evidence for Palaeolithic and Neolithic subsistence. Eur J Clin Nutr. 2002;56 (12):1270-8. Studies Consortium, 1(1), 3-10. Synthesis in higher education. EvoS Journal: The Journal of the Evolutionary.

Roff DA. Defining fitness in evolutionary models. Indian Academy of Sciences. 2008;87:339-48.

Roy TC, Springer BA, McNulty V, Butler NL. Physical fitness. Military Medicine. 2010;175,8:14, 14-20.

Sinclair DA. Toward a unified theory of caloric restriction and longevity regulation. Mech Ageing Dev. 2005;126:987-1002.

Tappeiner G, Tappeiner U, Walde J. Integrating disciplinary research into an interdisciplinary framework: a case study in sustainability research. Environ Model Assess. 2007;12:253-6. to Human Behavior: A Key to Survival in the Ivory Archipelago. Futures.

Varady KA, Hellerstein MK. Alternate-day fasting and chronic disease prevention: a review of human and animal trials. Am J Clin Nutr. 2007;86(1):7-13.

Williams GC, Nesse RM. The dawn of Darwinian medicine. Q Rev Biol. 1991;66:1-22.

Williams GC, Nesses RM. Why we get sick: the new science of Darwinian medicine. New York: Vintage Books; 1996.

Wilson DS. Evolution for everyone. New York: Delacorte Press; 2007. 